\title{
Application of Monte Carlo method in the simulation of circumference ratio
}

\author{
Zhao Hui Min ${ }^{1, a, *}$ \\ ${ }^{1}$ Bohai University, Jinzhou 121013, China \\ ahmzhao2008@163.com
}

Keywords: Monte Carlo method, Circumference ratio, Buffon's Needle

\begin{abstract}
We have calculated the circumference ratio by Monte Carlo method and Buffon's Needle algorithm. Our results reveal that the estimated value approaches to the expected value as the Monte Carlo steps increase. The error between the estimated and the expected values decreases inversely as the square root of MCS increases. When MCS reaches 1010, the error is only one part per million of the expected value.
\end{abstract}

\section{Introduction}

Monte Carlo method is a powerful simulation method based on random number and statistics [1]. As well known, many physical processes occur randomly in real world, e.g. the decay of nucleus, the transportation of particles in media as well as the Brown movement of molecules. In 1777, G.L.L. de Buffon, a famous mathematician, proposed the famous Needle Problem to calculate the circumference ratio. With the emergence of computer science, von Neumann, a famous mathematician, proposed a new method based on random number, to simulate the collisions between neutrons and nucleus which resulted in the nucleus reaction [2]. Von Neumann named this method Monte Carlo, which is the name of gambling city in Mexico. Monte Carlo method has many important applications in the teaching of simulation. For instance, Monte Carlo method can be used to simulate the problems of the transport of articles in media [2], magnetic phase transition [3,4,5], high-dimensional mathematic integral, engineering and economics. Random number is most frequently used in Monte Carlo simulations. In the simulations, random number must satisfy the following requirements, that is, it must be conveniently and quickly generated, and it must be homogeneous in distribution. Moreover, it should be independent and non-correlated. Convenience means that random number must be generated quickly and not consume much computing time. Homogeneous distribution means that random number should be distributed in the region homogenously. Independence means that random number must not be correlated. Only satisfying the above requirements, does random number work well in simulation. In this article, we employ Monte Carlo method to simulate the circumference ratio and study the convergence behavior of simulation with increasing Monte Carlo steps.

\section{Methods}

Random number is generated through certain mathematic expression. Usually, random number is generated via the following equation.

$$
\begin{aligned}
& x_{n+1}=\left(a x_{n}+c\right) \bmod (m) \\
& \xi_{n}=x_{n} / m
\end{aligned}
$$

In the above equation, $C=A \bmod (B)$ means the remainder generated in the operation of $\mathrm{A}$ divided by B. $x_{0}$ is the seed of random number. If one changes the seed of random number, then a new series of random number can be generated. $a, c, x_{0}$ and $m$ are the integers larger than zero, and are called multiplier, increment, initial value and module. 
The foundation of Monte Carlo simulation is the law of large numbers and central limit theorem. According to the law of large numbers, if one randomly samples $n$ numbers from the region [a,b] with homogenous probability and calculate the values of corresponding function $h\left(u_{i}\right)$, the average value of function should converge to the expected value through the following equation

$$
\lim _{n \rightarrow \infty} \frac{1}{n} \sum_{i=1}^{n} h\left(u_{i}\right)=\frac{1}{b-a} \int_{a}^{b} h(u) d u
$$

According to the law of larger numbers, if we sample enough function values, the estimated function value should converge to the expected value.

The central limit theorem tells that no matter how the random variable is distributed, the summation of total random variables must satisfy the canonical distribution, that is,

$$
f(x)=\frac{1}{\sqrt{2 \pi} \sigma} e^{-(x-\mu)^{2} / 2 \sigma^{2}}
$$

In the above equation, $\mu$ is the expected value and $\sigma^{2}$ is variance. According to the central limit theorem, the difference between expected value and estimated value should satisfy the following equation

$$
\left|I_{n}-\mu\right|<\lambda \frac{\sigma}{\sqrt{N}}
$$

According to the central limit theorem, the error between the estimated value and the expected value should inversely decrease as $\sqrt{N}$ increases.

\section{Simulation results}

According to the Buffon's Needle Problem, the circumference ratio can be calculated through the following way. As shown in Fig. 1, the needles are thrown with equal probability and they cover the square homogeneously. If we count the numbers of needles fallen in the quarter of circle, we can get the area ratio of the quarter circle to the square.

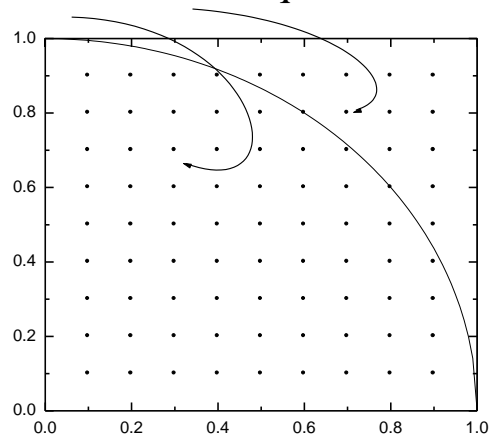

Fig. 1: Scheme of the Needle Problem. The black points are the needles thrown with equal probability. The black arc is the quarter circle.

The Needle Problem can be realized via the following algorithm. (i) A pair of random numbers $\left(x_{n}, y_{n}\right)$ are generated, with $x_{n} \in(0,1), y_{n} \in(0,1)$. (ii) The value of $x_{n}^{2}+y_{n}^{2}$ is calculated. If the value is less than 1.0, the needle point fell into the arc and the count of needle fallen into the arc increases one. (iii) The total number of needles $n$ fallen into the arc is calculated and the ratio of needles fallen into the arc to the total thrown needle satisfies $\lim _{N \rightarrow \infty} \frac{n}{N}=\frac{\pi}{4}$. Our simulation results are listed in table 1 . 
Table 1: The estimated value of circumference ratio for increasing Monte Carlo steps.

\begin{tabular}{|c|c|c|c|c|c|c|}
\hline$N$ & $10^{3}$ & $10^{4}$ & $10^{5}$ & $10^{6}$ & $10^{7}$ & $10^{8}$ \\
\hline$\pi$ (1) & 3.0720 & $0^{3.1756}$ & $2^{3.1359}$ & $4^{3.1436}$ & $\begin{array}{l}3.1402 \\
1\end{array}$ & $7^{3.1414}$ \\
\hline$\pi$ (2) & 3.2000 & $0^{3.1492}$ & $2^{3.1407}$ & $1 \begin{array}{l}3.1402 \\
\end{array}$ & $3^{3.1419}$ & $8^{3.1413}$ \\
\hline$\pi$ (3) & 3.0720 & $0^{3.1728}$ & $8^{3.1418}$ & $4^{3.1411}$ & $2^{3.1416}$ & $5^{3.1418}$ \\
\hline$\pi$ (4) & 3.1240 & $\begin{array}{ll} & 3.1660 \\
0 & \end{array}$ & $8^{3.1410}$ & $8^{3.1428}$ & $8^{3.1416}$ & $5^{3.1415}$ \\
\hline$\pi(5)$ & 3.1520 & $0^{3.1320}$ & $2^{3.1363}$ & $0^{3.1407}$ & $7^{3.1415}$ & $3^{3.1415}$ \\
\hline$\pi \quad(6)$ & 3.0520 & $0^{3.1292}$ & $8^{3.1404}$ & $6^{3.1405}$ & $1^{3.1418}$ & $7^{3.1415}$ \\
\hline$\pi \quad(7)$ & 3.1800 & $0^{3.1208}$ & $6^{3.1361}$ & $4^{3.1435}$ & 13.1410 & $1^{3.1416}$ \\
\hline$\pi(8)$ & 3.1080 & $0^{3.1516}$ & $4^{3.1374}$ & $5^{3.1465}$ & $8^{3.1419}$ & $9^{3.1413}$ \\
\hline$\pi$ (9) & 3.0960 & $0^{3.1492}$ & $0^{3.1374}$ & $5^{3.1382}$ & $8^{3.1411}$ & $6^{3.1415}$ \\
\hline$\pi(10)$ & 3.0640 & $0^{3.1488}$ & $6^{3.1381}$ & $2^{3.1431}$ & $2^{3.1425}$ & $\begin{array}{ll} & 3.1418 \\
5 & \end{array}$ \\
\hline$\overline{\bar{\pi}}$ & 3.1120 & $2^{3.1495}$ & $56^{3.1385}$ & 3.1420 & 3.1415 & $75^{3.1415}$ \\
\hline
\end{tabular}

It is noticed that the estimated value of circumference ratio approaches to the expected value as the Monte Carlo steps (MCS) increases. When MCS reaches $10^{9}$, the estimated value gets to 3.141588. For MCS of $10^{10}$, the estimated value reaches 3.1415974, which is very close to the expected value 3.1415926 .

The error of estimated value versus MCS is shown in Fig. 2. It is noticed that the error decreases inversely as $\sqrt{N}$ increases. We fit the data points by power law $y=a / \sqrt{N}$ and find the parameter $a=0.923$.

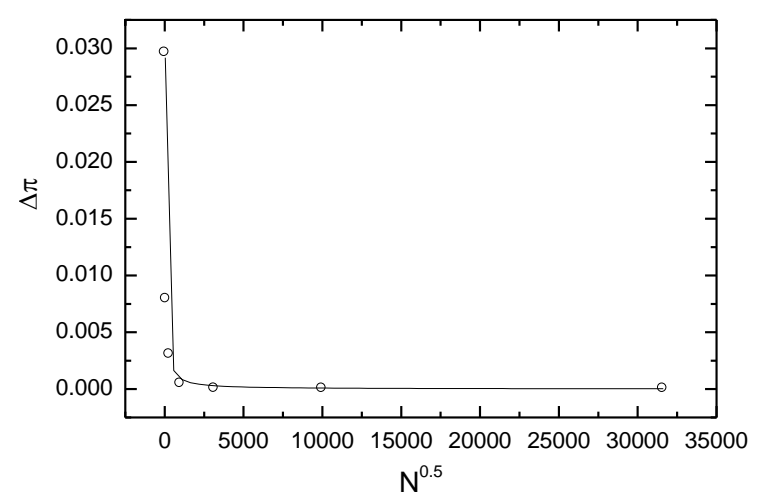

Fig. 2: Simulation error versus $\sqrt{N}$. The circles are the data points of error. The curve is the theoretical fit.

\section{Conclusions}

We calculate the circumference ratio by Monte Carlo method and Buffon's Needle scheme. Our results reveal that the estimated value approaches to the expected value as MCS increases. The error 
of estimated value inversely decreases as the square root of MCS increases. When MCS reaches to $10^{10}$, the error of estimated value is one part per million of the expected value.

\section{Acknowledgment}

This work is supported by Social Science Foundation of Liaoning Province (No. L15DWW005) and Bohai University Teaching Reform Project (No.BDJG15QNB002).

\section{References}

[1] J.V. Bradley, Distribution Free Statistical Tests, New York: Prentice Hall, 1968.

[2] L.L. Carter, E.D. Cashwell, Particle Transport Simulation with the Monte Carlo Methods, Oak Ridge, TN: USERDA Technical Information Center, 1975.

[3] K.C. Zhang, P.-Y. Song, Spin Glass Dynamics in RKKY Interacting Disordered Magnetic System, Chin. Phys. B , 19 (2010) 097105.

[4] K. Binder, In Phase Transitions and Critical Phenomena, in: C. Domb, M.S. Green (Eds.), Academic, New York, 1976.

[5] D.P. Laudau, K. Binder, A Guide to Monte Carlo Simulations in Statistical Physics, Cambridge University Press, 2008, pp.78-79 